\title{
Review of: "SARS-CoV-2 infection risk during delivery of childhood vaccination campaigns: a modelling study"
}

elena bozzola

Potential competing interests: The author(s) declared that no potential competing interests exist.

Procter et al. focused on an important topic, such as childhood vaccine campaigns during COVID 19 pandemics.

As previous studies underlined, routine immunization activities had been disrupted either by vaccine centers closure either by parents' decisions all over the world. We faced a consequent reduction in routine immunization coverage, leading to a serious life-threating problem for unvaccinated or under-vaccinated children. An Italian survey revealed that most parents were reluctant to get their children vaccinated because of lack of information, such as availability of booking child's vaccination appointment and awareness of preventive adopted measures, or because they fear infection with the COVID-19 virus. (doi: 10.1186/s13052-021-01023-6)

Effective personal protective equipment, hygiene measures, symptomatic screening and appropriate campaign timing may reduce COVID-29 infection risks to both health care providers and families. Procter et al's conclusion should be strength in order to encourage people not to skip routine vaccination appointment in case of new pandemics. Of note, the study should be of interest to health care providers in order to optimize the organization of their daily activities. In the last months, due to COVID-19 pandemic, pediatricians have observed a significant drop of in-person child health visits, vaccinations and physical-neurodevelopment screenings that may endanger children's health. The Italian Pediatric Society strongly supports and emphasizes the importance of routine well childcare assistance and immunization, suggesting scheduling sick visits and well-child visits during different times of the day and/or in different setting, promoting hygienic strategies and the correct use of effective personal protective equipment. (doi: 10.1186/s13052-020-00899-0).

One variability that should be added to examine the risk of COVID 19 transmission is family education. Providing correct and timely information to prevent infection, by everyday protective measurements, as reported by WHO may influence the risk of infection spreading.

(https://www.who.int/emergencies/diseases/novel-coronavirus-2019/advice-for-public) For example, wearing a mask correctly and consistently especially when social distancing is not possible may optimize the protection. Mask is most effective when worn properly. As a consequence, uncovering nose or mouth, re-using single-use mask, hanging it from ears or placing it under chin may affect the effectiveness of personal protective equipment against COVID-19. (https://www.canada.ca/en/public- 
health/services/diseases/2019-novel-coronavirus-infection/prevention-risks/about-non-medical-masks-facecoverings.html) 